S. Takayama

Nagoya Math. J.

Vol. 146 (1997), 185-197

\title{
ON RELATIVE BASE POINT FREENESS OF ADJOINT BUNDLE
}

\author{
SHIGEHARU TAKAYAMA
}

\begin{abstract}
We give an effective result on the relative base point freeness of an adjoint bundle for a pair of a projective morphism and a relatively ample line bundle.
\end{abstract}

\section{$\S 1$. Introduction}

Recently, Angehrn and Siu [AS] and Tsuji [Tj] independently obtained results on the following:

FujitA'S FREENESS CONJECTURE OF ADJOINT BUNDLES. ([F]) Let $X$ be an $n$-dimensional projective manifold defined over $\mathbb{C}$ with an ample line bundle $L$. Then the adjoint bundle $\mathcal{O}_{X}\left(K_{X} \otimes L^{\otimes m}\right)$ is generated by global sections for every $m>n$.

Their effective bounds are $m>n(n+1) / 2$. The basic ideas of their proofs from [AS] and [Tj] (use of Riemann-Roch theorem, Nadel's vanishing theorem, Ohsawa-Takegoshi's $L^{2}$-extension theorem and so on) are extremely simple and can be applied to a variety of contexts. In this note we would like to go into detail about the method and consider the following relative version:

MaIn TheOREM. Let $f: X \longrightarrow Y$ be a projective morphism from a complex manifold $X$ to a complex space $Y$, and let $L$ be a relatively ample line bundle on $X$. Then $\mathcal{O}_{X}\left(K_{X} \otimes L^{\otimes m}\right)$ is $f$-free, i.e., the natural sheaf homomorphism

$$
f^{*} f_{*} \mathcal{O}_{X}\left(K_{X} \otimes L^{\otimes m}\right) \longrightarrow \mathcal{O}_{X}\left(K_{X} \otimes L^{\otimes m}\right) \quad \text { is surjective, }
$$

for every

$$
m>\frac{1}{2} d(d+1)
$$

here $d$ is the maximum dimension of the fibres of $f$.

Received September 20, 1995. 
Our relative version has some applications to the classification theory of higher dimensional algebraic varieties. For example, we have the following (refer to $[\mathrm{KMM}]$ for terminologies):

Corollary. Let $X$ be a projective manifold defined over $\mathbb{C}$ and let $\varphi: X \longrightarrow X^{\prime}$ be the contraction morphism of an extremal ray $R$ of $\overline{N E}(X)$. Then the $d(d+1) / 2$-th anti-pluri-canonical divisor $-d(d+1) / 2 K_{X}$ is $\varphi$-free, here $d$ is the maximum dimension of the fibres of $\varphi$.

This corollary is much helpful to the classification of the singular fibres of $\varphi$ and contraction morphisms (cf. $[\mathrm{K}]$ )

The reader should refer to [D2] (analytic approach) and [L] (algebraic approach) for the recent development of the theory of adjoint bundles.

I would like to express my thanks to Professor Takeo Ohsawa for his suggestions and encouragement during the preparation of this paper. I would also like to express my thanks to Professor Hajime Tsuji who kindly explained his basic ideas.

\section{$\S 2$. Singular Hermitian metric and vanishing theorem}

Our basic tool is singular Hermitian metrics as in [D1], [D2]. We use vector bundles and the associated locally free sheaves interchangeably. In this section we let $X$ be a complex manifold.

\section{A. Singular Hermitian metric}

Let $L$ be a holomorphic line bundle on $X$. A metric $h$ on $L$ is called singular Hermitian, if there exist a function $\varphi \in L_{\text {loc }}^{1}(X)$ and a smooth Hermitian metric $h_{0}$ on $L$ such that $h=e^{-\varphi} h_{0}$ holds. This defines a closed current

$$
\operatorname{curv} h:=\operatorname{curv} h_{0}+\sqrt{-1} \partial \bar{\partial} \varphi
$$

where curv $h_{0}$ is the curvature form of the Hermitian metric $h_{0}$ and $\partial \bar{\partial}$ is taken in the sense of currents. The $(1,1)$-current curv $h$ is said to be the curvature current of the singular Hermitian line bundle $(L, h)$. It is easy to see that curv $h$ is independent of the choices of $h_{0}$ and $\varphi \cdot \sqrt{-1} \bar{\partial} \partial \log h$ is the formal expression of curv $h$. For a singular Hermitian line bundle $(L, h)$ on a Hermitian manifold $(X, \omega)$. The $L^{2}$-sheaf $\mathcal{L}^{2}(L, h)$ is the sheaf defined by

$$
\mathcal{L}^{2}(L, h)(U)=\left\{s \in \Gamma(U, L) ; h_{0}(s, s) e^{-\varphi} \in L_{\mathrm{loc}}^{1}(U)\right\},
$$


where $h=e^{-\varphi} h_{0}$ is a local expression of $h$ as above. Similarly, the multiplier ideal sheaf $\mathcal{I}(h)$ of the singular Hermitian metric is defined by

$$
\mathcal{I}(h)(U):=\left\{f \in \Gamma\left(U, \mathcal{O}_{M}\right) ;|f|^{2} e^{-\varphi} \in L_{\text {loc }}^{1}(U)\right\} .
$$

These sheaves do not depend of the choices $\varphi, h_{0}$ and $\omega$, and satisfy the following relation: $\mathcal{L}^{2}(L, h)=L \otimes \mathcal{I}(h)$.

\section{B. Vanishing theorem}

We recall the following:

NADEL'S COHERENCE AND VANISHING THEOREM 2.1. ([N], $[\mathrm{D} 1, \S 4])$ Let $(X, \omega)$ be a complete Kähler manifold and let $(L, h)$ be a singular Hermitian line bundle on $X$. Assume that there exists a real number $c$ such that curv $h \geq c \omega$ on $X$. Then

(1) the sheaf $\mathcal{I}(h)$ is a coherent ideal sheaf of $\mathcal{O}_{X}$, and

(2) if $c$ is positive, the $q$-th $L^{2}$-cohomology group

$$
H_{(2)}^{q}\left(X, K_{X} \otimes L \otimes \mathcal{I}(h)\right)=0
$$

for every $q \geq 1$.

As a simple application of the above theorem, we have

Proposition 2.2. Let $(X, \omega)$ be a complete Kähler manifold, $x$ be a point of $X$, and let $L$ be a holomorphic line bundle on $X$. Assume that $L$ admits a singular Hermitian metric $h_{x}$ such that

(1) there exists a positive constant $c$ such that curv $h_{x} \geq c \omega$, and that

(2) $x$ is isolated in the zero complex space $V \mathcal{I}\left(h_{x}\right)$.

Then there exists a holomorphic section of $K_{X} \otimes L$ which does not vanish at $x$.

We will need the following Serre type vanishing theorem:

Proposition 2.3. ([F, Theorem $\left.\mathrm{N}^{\prime}\right]$ ) Let $L$ be a positive line bundle on a weakly 1-complete manifold $X$, i.e., a complex manifold with a smooth plurisubharmonic exhaustion function $\Phi: X \longrightarrow \mathbb{R}$. Then for every coherent analytic sheaf $\mathcal{F}$ on $X$ and for every $c<\sup _{X} \Phi$, there exists a positive integer $m_{0}$ such that

$$
H^{q}\left(X_{c}, \mathcal{F} \otimes L^{\otimes m}\right)=0
$$

for any $q \geq 1$ and for any $m \geq m_{0}$, where $X_{c}:=\{x \in X ; \Phi(x)<c\}$ is a sublevel set of $(X, \Phi)$. 


\section{C. Singular Hermitian metric with analytic singularities} [AS, §2]

In this subsection we explain how to construct a singular Hermitian metric and how to control the multiplier ideal sheaf. The standard method of the construction is to use holomorphic sections; such metrics are said to be singular Hermitian metrics with analytic singularities.

It is convenient to introduce the notion of rational coefficient geometry as follows. We consider a family of local holomorphic functions $s=\left\{s_{\lambda} ; s_{\lambda} \in\right.$ $\left.H^{0}\left(U_{\lambda}, \mathcal{O}_{X}\right)\right\}_{\lambda \in \Lambda}$ for some locally finite open cover $\left\{U_{\lambda}\right\}_{\lambda \in \Lambda}$ of $X$. For a positive rational number $q$ and for any smooth Hermitian holomorphic line bundle $\left(L, h_{0}\right)$ on $X$, the family of local holomorphic functions $s$ is said to be a multivalued holomorphic section of $L^{\otimes q}$ over $X$, if there exists a positive integer $p$ such that $p q$ being an integer and that $s^{p}:=\left\{s_{\lambda}^{p}\right\}_{\lambda \in \Lambda}$ defines an element of $H^{0}\left(X, L^{\otimes p q}\right)$. We denote

$$
\begin{aligned}
|s|:=\left(h_{0}^{\otimes p q}\left(s^{p}, s^{p}\right)\right)^{1 /(2 p)}: & \text { the pointwise length, } \\
(s)_{0}:=\left\{x \in X ; s_{\lambda}(x)=0\right. & \text { for some } \lambda \in \Lambda\} .
\end{aligned}
$$

We just consider $(s)_{0}$ as a set of zeros. We also define a singular Hermitian metric $h$ of $L^{\otimes q}$ by a family of local real valued measurable functions such that $h^{p}$ defines a singular Hermitian metric of $L^{\otimes p q}$. We can also define the curvature current and the multiplier ideal sheaf.

Let $s_{1}, \ldots, s_{k}$ be a finite number of multivalued holomorphic sections $L^{\otimes q}$ such that $\left(s_{i}\right)^{p}(1 \leq i \leq k)$ is a holomorphic section of $L^{\otimes p q}$ for some positive integer $p$ with $p q$ being an integer. Then we can define a singular Hermitian metric of $L^{\otimes q}$ by

$$
h:=\frac{h_{0}^{q}}{\sum_{i=1}^{k}\left|s_{i}\right|^{2}} .
$$

The curvature current is a closed positive current on $X$. Indeed, for local expressions $s_{i}=\left\{s_{i \lambda}\right\}_{\lambda \in \Lambda}$, we see

$$
\operatorname{curv} h=\sqrt{-1} \partial \bar{\partial} \log \sum_{i=1}^{k}\left|s_{i \lambda}\right|^{2}
$$

on every open set $U_{\lambda}$. We note that both the positivity of the curvature current and the multiplier ideal sheaf do not depend on the smooth Hermitian metric $h_{0}$. Let $\mathcal{J}$ be the sheaf of ideal of $\mathcal{O}_{X}$ generated locally by 
$\left\{\left(s_{i}\right)^{p}\right\}_{i=1}^{k}$. We assume that the support of $\mathcal{O}_{X} / \mathcal{J}$ is compact. We take a modification $\pi: \tilde{X} \longrightarrow X$ by a finite number of successive monoidal transforms with nonsingular centers and a family of smooth divisors $E_{i}$ in $\tilde{X}$ with only simple normal crossing so that the following three consitions hold:

(0) For every $i, \pi\left(E_{i}\right) \subset \operatorname{supp} \mathcal{O}_{X} / \mathcal{J}$.

(1) The sheaf $\pi^{-1} \mathcal{J} \cdot \mathcal{O}_{\widetilde{X}}$ which is the image of $\pi^{*} \mathcal{J}$ under the natural map $\pi^{*} \mathcal{J} \longrightarrow \mathcal{O}_{\tilde{X}}$ is equal to the ideal sheaf $\mathcal{O}\left(-\sum r_{i}^{\prime} E_{i}\right)$ for some non-negative integers $r_{i}^{\prime}$.

(2) $K_{\widetilde{X}}=\pi^{*} K_{X} \otimes \mathcal{O}\left(\sum b_{i} E_{i}\right)$ for some non-negative integers $b_{i}$. In other words, the holomorphic Jacobian determinant of the map $\pi: \tilde{X} \longrightarrow X$ vanishes precisely of order $b_{i}$ along $E_{i}$ and vanishes nowhere on $\tilde{X}-\bigcup_{i} E_{i}$. Let $r_{i}:=r_{i}^{\prime} / p$. For every $t \geq 0$, we set

$$
\mathcal{I}(t):=\mathcal{L}^{2}\left(\mathcal{O}_{X},\left(\sum_{i=1}^{k}\left|s_{i}\right|^{2}\right)^{-t}\right) .
$$

We see that every $\mathcal{I}(t)$ is a coherent ideal sheaf by $2.1(1)$ and that $\mathcal{I}(1)=$ $\mathcal{I}(h)$. Then a point $x$ on $X$ belongs to the zero complex subspace $V \mathcal{I}(t)$ if and only if there exists an index $i$ such that $E_{i}$ intersects $\pi^{-1}(x)$ and that $t r_{i}-b_{i} \geq 1$. For every point $x \in V \mathcal{I}(1)$, we see that

$$
\begin{aligned}
\sup \{t & \left.\geq 0 ; \mathcal{I}(t)_{x}=\mathcal{O}_{X, x}\right\} \\
& =\min \left\{t \geq 0 ; t r_{i}-b_{i} \geq 1 \text { for } i \text { such that } E_{i} \text { intersects } \pi^{-1}(x)\right\} .
\end{aligned}
$$

Note that the quantity, say $\alpha(x)$, is always a rational number and $0<$ $\alpha(x) \leq 1$.

\section{$\S 3$. Preliminary lemma}

\section{A. Reduction and non-vanishing with a parameter space}

Let $f: X \longrightarrow Y$ and $L$ be as in Main Theorem. We fix a point $x$ on $X$. The situation is local on $Y$, so we may assume that $Y$ is a closed complex subspace of the unit ball $\mathbb{B}^{M}$ in $\mathbb{C}^{M}$ with the global coordinate $\left(y_{1}, \ldots, y_{M}\right)$ and that $f(x)=0$. Since $L$ is $f$-ample, restricting $Y$ on a smaller ball $\mathbb{B}^{M}$ if necessary, we may assume $L$ admits a smooth Hermitian metric $h$ whose curvature form is positive on $X$.

In the local situation as above, we show the following non-vanishing lemma with a parameter space. It is important to handle the case that the zero complex subspace of the multiplier ideal sheaf has singularities. 
LEMMA 3.1. ([AS, Lemma 4.1]) Let $Z$ be a closed subvariety (reduced and irreducible) in $X$ of positive dimension $d$ such that $x \in Z$ and $f(Z)=$ $f(x), B_{0}$ and $B_{1}$ be smaller balls centered at $0 \in \mathbb{B}^{M}$ with $B_{0} \varsubsetneqq B_{1} \varsubsetneqq \mathbb{B}^{M}$, $Y_{i}:=Y \cap B_{i}$ and $X_{i}:=f^{-1}\left(Y_{i}\right)$ for $i=0$, 1, and let $N$ be a positive integer. Let $\Delta^{\prime}$ be a local holomorphic curve in $Z$ passing through $x$ with $x$ as the only singularity such that the normalization $\sigma: \Delta \longrightarrow \Delta^{\prime}$ is a one to one holomorphic map from the open unit disk $\Delta$ in $\mathbb{C}$ with $\sigma(0)=x$. Then, replace $\Delta$ with a smaller disk if necessary, there exist a positive integer $m$ and a finite number of holomorphic sections $\left\{\widetilde{\tau}_{j}\right\}_{j=1}^{K} \subset H^{0}\left(X_{1} \times\right.$ $\left.\Delta, p r_{X}^{*} L^{\otimes m(N+1)}\right)$, where $p r_{X}: X \times \Delta \rightarrow X$ is the first projection, such that

$$
\left.\widetilde{\tau}_{j}\right|_{Z \times u} \in H^{0}\left(Z, L^{\otimes m(N+1)} \otimes \mathcal{M}_{Z, \sigma(u)}^{m N}\right) \quad \text { for every } u \in \Delta-0
$$

and for every $j$, and that their common zeros satisty

$$
x \in X_{0} \cap \bigcap_{j=1}^{K}\left(\left.\widetilde{\tau}_{j}\right|_{X_{1} \times 0}\right)_{0} \varsubsetneqq Z .
$$

Proof. We denote the ideal sheaf of the graph $\sigma \times 1: \Delta \longrightarrow Z \times \Delta$ by $\mathcal{I}_{\Gamma} \subset \mathcal{O}_{Z \times \Delta}$. Since $Z$ is compact, the direct image sheaf $p r_{\Delta *}\left(p r_{X}^{*} L^{\otimes m(N+1)}\right.$ $\left.\otimes \mathcal{O}_{Z \times \Delta} \otimes \mathcal{I}_{\Gamma}^{m N}\right)$ is a coherent sheaf on $\Delta$, where $p r_{\Delta}: Z \times \Delta \longrightarrow \Delta$ be the projection. The sheaf $p r_{\Delta_{*}}\left(p r_{X}^{*} L^{\otimes m(N+1)} \otimes \mathcal{O}_{Z \times \Delta} \otimes \mathcal{I}_{\Gamma}^{m N}\right)$ is generically locally free with $H^{0}\left(Z \times u, L^{\otimes m(N+1)} \otimes \mathcal{M}_{Z \times u, \sigma(u) \times u}^{m N}\right)$ as the generic fibre. We see that the latter space is non-zero for every large $m$ and for every $u \in \Delta-0$ by the following asymptotic dimension compairing:

$$
\begin{aligned}
\operatorname{dim} H^{0}\left(Z, L^{\otimes m(N+1)}\right) & =(N+1)^{d}\left(L^{d} \cdot Z\right)(d !)^{-1} m^{d}+O\left(m^{d-1}\right) ; \\
\operatorname{rank} \mathcal{O}_{Z} / \mathcal{M}_{Z, \sigma(u)}^{m N} & =\left(\begin{array}{c}
m N+d-1 \\
d
\end{array}\right)=N^{d}(d !)^{-1} m^{d}+O\left(m^{d-1}\right) .
\end{aligned}
$$

Then by Theorem A of Cartan-Serre we see that, for every large $m$, there exists a section $\widetilde{\tau} \in H^{0}\left(Z \times \Delta, p r_{X}^{*} L^{\otimes m(N+1)} \otimes \mathcal{O}_{Z \times \Delta} \otimes \mathcal{I}_{\Gamma}^{m N}\right)$ such that $\left.\widetilde{\tau}\right|_{Z \times 0}$ is not identically zero.

For a smaller disk $\Delta_{1}$, we take a sublevel set $W$ of a weakly 1-complete manifold $X \times \Delta$ for an appropriate smooth plurisubharmonic exhaustion function which contains $X_{1} \times \Delta_{1}$. By Proposition 2.3, for every $x_{0} \times u_{0} \in$ $X \times \Delta-Z \times \Delta$, there exists a positive integer $m_{0}$ such that

$$
H^{1}\left(W, p r_{X}^{*} L^{\otimes m} \otimes \mathcal{I}_{Z \times \Delta} \otimes \mathcal{M}_{X \times \Delta, x_{0} \times u_{0}}\right)=0
$$

for any $m \geq m_{0}$. Hence we can extend $\widetilde{\tau}$ as sections $\widetilde{\tau}_{1}, \ldots, \widetilde{\tau}_{K} \in H^{0}\left(X_{1} \times\right.$ $\left.\Delta_{1}, p r_{X}^{*} L^{\otimes m(N+1)}\right)$ which satisfy the desired properties. 


\section{B. Calculus Lemma}

The following simple calculus lemma on non-integrability will be used later to locate the zero-set of the multiplier ideal sheaf of a singular metric.

LEMMA 3.2. ([AS, Lemma 3.1]) Let $m$ and $N$ be positive integers and $0<a<1$. Let $f_{1}, \ldots, f_{k}$ be holomorphic functions on the unit polydisk $\Delta^{n}$ on $\mathbb{C}^{n}$ with coordinates $z, w_{1}, \ldots, w_{n-1}$. Let $H:=\{z=0\}$ and let $V$ be the subset of $H \cap \Delta^{n}$ where the vanishing order of $\left.f_{j}\right|_{H \cap \Delta^{n}}$ is at least $m N$ for any $j$. Let $d$ be the codimension of $V$ in $H \cap \Delta^{n}$ at the origin. Then $|z|^{-2 a}\left(\sum\left|f_{j}\right|^{2}\right)^{-t /(m N)}$ is not locally integrable at the origin for $t \geq d+m N(1-a)$.

Proof. By slicing and Fubini's theorem, we may assume $d=n-1$. Then $\sum\left|f_{j}\right|^{2} \leq C_{1}\left(|z|^{2}+|w|^{2 m N}\right)$ for some positive constant $C_{1}$, where we set $|w|^{2}:=\sum_{i=1}^{n-1}\left|w_{i}\right|^{2}$. The non-integrability of $|z|^{-2 a}\left(\sum\left|f_{j}\right|^{2}\right)^{-t /(m N)}$ follows from that of $|z|^{-2 a}\left(|z|^{2}+|w|^{2 m N}\right)^{-t /(m N)}$. Then we see the nonintegability by direct calculation by using polar coordinates for $z$ and $w$ with $x=|z|^{2}$ and $y=|w|$.

\section{$\S 4$. Proof of Theorem}

We fix a point $x$ on $X$. We let $f: X \longrightarrow Y \subset \mathbb{B}^{\mathbb{M}} \subset\left(\mathbb{C}^{M} ; y_{1}, \ldots, y_{M}\right)$ and $(L, h)$ be the local reduction around $f(x)$ as in $3 \mathrm{~A}$. We take smaller balls $B_{0}$ and $B_{1}$ centered at $0 \in \mathbb{B}^{M}$ with $B_{0} \varsubsetneqq B_{1} \varsubsetneqq \mathbb{B}^{M}$. We set $Y_{i}:=Y \cap B_{i}$ and $X_{i}:=f^{-1}\left(Y_{i}\right)$ for $i=0,1$. With these notations, our Main Theorem follows from the following

ThEOREM 4.1. For every $m>d_{0}\left(d_{0}+1\right) / 2$, there exists a holomorphic section $\tau \in H^{0}\left(X_{1}, \mathcal{O}_{X}\left(K_{X} \otimes L^{\otimes m}\right)\right)$ such that $\tau(x) \neq 0$, where $d_{0}$ is the dimension of a maximum dimensional irreducible component of the fibre $f^{-1}(f(x))$ which contains $x$.

By Proposition 2.2, all we have to do is to show the following

Proposition 4.2. For every $m>d_{0}\left(d_{0}+1\right) / 2,\left.L\right|_{X_{1}} ^{\otimes m}$ admits a singular Hermitian metric $H_{m}$ such that

(1) the curvature current dominants a complete Kähler form on $X_{1}$, and that

(2) $x$ is isolated in the zero subspace $V \mathcal{I}\left(H_{m}\right)$.

If $f$ is constant, then $X$ is a projective manifold with an ample line bundle $L$, that is (a part of) the statement of [AS], [Tj]. Hence we assume that $f$ is non-constant. 


\section{A. Statement of the induction step}

We formulate an induction statement for the proof of Proposition 4.2. Let $m_{d}:=\sum_{n=d+1}^{d_{0}} n$ for $0 \leq d<d_{0}$ and let $m_{d_{0}}:=0$. We take rational numbers $0=\varepsilon\left(d_{0}+1\right)<\varepsilon\left(d_{0}\right)<\varepsilon\left(d_{0}-1\right) \cdots<\varepsilon(0)<1$. For every positive rational number $q$ and for every multivalued holomorphic section $s$ of $L^{\otimes q}$ on an open set of $X$, we denote $|s|$ the length with respect to the smooth Hermitian metric $h$. For every $d$ with $0 \leq d \leq d_{0}$, we consider the following

Induction Statement $(*)_{d}$. There exist a rational number $\varepsilon(d+$ 1) $<\varepsilon_{d}<\varepsilon(d)$ and a finite number of multivalued holomorphic sections $s_{1}^{(d)}, \ldots, s_{k_{d}}^{(d)}$ of $L^{\otimes\left(m_{d}+\varepsilon_{d}\right)}$ on $X_{1}$ such that

(i) $\left(\cap_{i=1}^{k_{d}}\left(s_{i}^{(d)}\right)_{0} \cap X_{0}\right) \subset f^{-1}(f(x))$,

(ii) $x \in\left(Z_{d}(1) \cap X_{0}\right) \subset f^{-1}(f(x))$,

(iii) $x \notin Z_{d}(t)$ for $t<1$, and that

(iv) The dimension of $Z_{d}(1)$ at $x$ is at most $d$, where

$$
\mathcal{I}_{d}(t):=\mathcal{L}^{2}\left(\mathcal{O}_{X_{1}},\left(\sum_{i=1}^{k_{d}}\left|s_{i}^{(d)}\right|^{2}\right)^{-t}\right) \quad \text { for every } t \geq 0
$$

be the multiplier ideal sheaf and where $Z_{d}(t):=V \mathcal{I}_{d}(t)$ be the complex subspace of $X_{1}$ defined by the ideal sheaf $\mathcal{I}_{d}(t)$.

We note that, by the vanishing theorem: Proposition 2.3, there exist a finite number of multivalued holomorphic sections $\left\{t_{i}\right\}_{i=1}^{K}$ of $L$ on $X_{1}$ such that $X_{0} \cap \bigcap_{i=1}^{K}\left(t_{i}\right)_{0}$ is empty. We verify the first step:

LEMMA 4.3. $(*)_{d_{0}}$ holds.

Proof. We set

$$
\begin{aligned}
\mathcal{I}_{*}(t) & :=\mathcal{L}^{2}\left(\mathcal{O}_{X},\left(\sum_{i=1}^{M}\left|f^{*} y_{i}\right|^{2}\right)^{-t}\right) \quad \text { for every } t \geq 0 ; \\
\alpha_{*} & :=\sup \left\{t \geq 0 ; \mathcal{I}_{*}(t)_{x}=\mathcal{O}_{X, x}\right\} .
\end{aligned}
$$

We see that every $\mathcal{I}_{*}(t)$ is a coherent ideal sheaf and that $\alpha_{*}$ is a positive rational number. We consider the complex subspace $V \mathcal{I}_{*}\left(\alpha_{*}\right)$ defined by the ideal sheaf $\mathcal{I}_{*}\left(\alpha_{*}\right)$. This space $V \mathcal{I}_{*}\left(\alpha_{*}\right)$ is compact and $x \in V \mathcal{I}_{*}\left(\alpha_{*}\right) \subset$ $f^{-1}(f(x))$. We choose a positive rational number $0<\varepsilon_{d_{0}}<\varepsilon\left(d_{0}\right)$ and set the multivalued holomorphic sections

$$
\left\{s_{i}^{\left(d_{0}\right)}\right\}_{i=1}^{k_{d_{0}}}:=\left\{f^{*} y_{i}^{\alpha_{*}} \times t_{j}^{\varepsilon_{d_{0}}}\right\}_{i, j}
$$


of $L^{\otimes \varepsilon_{d_{0}}}$ on $X_{1}$. Then we can verify $(*)_{d_{0}}$ by the following relation on $X_{0}$ :

$$
\mathcal{I}_{d_{0}}(t):=\mathcal{L}^{2}\left(\mathcal{O}_{X_{1}},\left(\sum_{i=1}^{k_{d_{0}}}\left|s_{i}^{\left(d_{0}\right)}\right|^{2}\right)^{-t}\right)=\mathcal{I}_{*}\left(t \alpha_{*}\right)
$$

\section{B. Concentration of the singularity}

In this subsection we verify the induction step. We assume $(*)_{d}$ with $d>0$. Let $p$ be a positive integer such that $p\left(m_{d}+\varepsilon_{d}\right)$ being integer and that $\left(s_{i}^{(d)}\right)^{p}\left(1 \leq i \leq k_{d}\right)$ is a holomorphic section of $L^{\otimes p\left(m_{d}+\varepsilon_{d}\right)}$ on $X_{1}$. Let $\mathcal{J}^{\prime}$ be the sheaf of ideal of $\mathcal{O}_{X_{0}}$ generated locally by $\left\{\left.\left(s_{i}^{(d)}\right)^{p}\right|_{X_{0}}\right\}_{i=1}^{k_{d}}$. By the assumption $(*)_{d}(\mathrm{i})$, we can extend $\mathcal{J}^{\prime}$ as a coherent ideal sheaf $\mathcal{J}$ of $\mathcal{O}_{X}$ by setting $\mathcal{J}=\mathcal{O}_{X}$ on $X-X_{0}$. We take a modification $\pi: \tilde{X} \longrightarrow X$ by a finite number of successive monoidal transforms with nonsingular centers and a family of smooth divisors $E_{i}$ in $\tilde{X}$ with only simple normal crossing so that the following three consitions hold:

(0) $\pi\left(E_{i}\right) \subset f^{-1}(f(x))$ for every $i$.

(1) The sheaf $\pi^{-1} \mathcal{J} \cdot \mathcal{O}_{\widetilde{X}}$ which is the image of $\pi^{*} \mathcal{J}$ under the natural map $\pi^{*} \mathcal{J} \longrightarrow \mathcal{O}_{\tilde{X}}$ is equal to the ideal sheaf $\mathcal{O}\left(-\sum r_{i}^{\prime} E_{i}\right)$ for some non-negative integers $r_{i}^{\prime}$.

(2) $K_{\widetilde{X}}=\pi^{*} K_{X} \otimes \mathcal{O}\left(\sum b_{i} E_{i}\right)$ for some non-negative integers $b_{i}$.

Let $r_{i}:=r_{i}^{\prime} / p$. The three conditions (ii)-(iv) in the statement $(*)_{d}$ can now be rewritten as the condition (ii) $)^{\prime}-(\text { iv) })^{\prime}$ below. Let $\Lambda$ be the set of all $i$ so that $E_{i}$ intersects $\pi^{-1}(x)$ and $r_{i}-b_{i} \geq 1$.

(ii) ${ }^{\prime} \Lambda$ is not empty;

(iii) ${ }^{\prime} i \in \Lambda$ then $r_{i}-b_{i}=1$;

(iv)' $i \in \Lambda$ then $\operatorname{dim} \pi\left(E_{i}\right) \leq d$.

We may assume that the index $i=0$ is an element of $\Lambda$ and that $\operatorname{dim} \pi\left(E_{0}\right)$ $=\max \left\{\operatorname{dim} \pi\left(E_{i}\right) ; i \in \Lambda\right\}$.

We choose $\varepsilon_{d-1}$ such that $\varepsilon(d)<\varepsilon_{d-1}<\varepsilon(d-1)$. Let $Z:=\pi\left(E_{0}\right) \subset$ $f^{-1}(f(x))$. If the dimension of $Z$ is less than $d$, then for $(*)_{d-1}$ we simply choose multivalued holomorphic sections

$$
\left\{s_{i}^{(d-1)}\right\}_{i=1}^{k_{d-1}}:=\left\{s_{i}^{(d)} \times t_{j}^{\left(m_{d-1}+\varepsilon_{d-1}\right)-\left(m_{d}+\varepsilon_{d}\right)}\right\}_{i, j}
$$

of $L^{\otimes\left(m_{d-1}+\varepsilon_{d-1}\right)}$ on $X_{1}$. We now assume without loss of generality that the dimension of $Z$ is precisely $d$. 
We take a local smooth holomorphic curve $\Gamma$ in $E_{0}-\bigcup\left\{E_{i} ; i \notin \Lambda\right\}$ with the following three properties:

(3) $\Gamma$ intersects $\pi^{-1}(x)$ at one point.

(4) $\Gamma$ either does not intersect $\bigcup_{i \neq 0} E_{i}$ or intersects $\bigcup_{i \neq 0} E_{i}$ only at the point $\Gamma \cap \pi^{-1}(x)$.

(5) $\Gamma$ either does not intersect $\pi^{-1}(\operatorname{Sing} Z)$ or intersects $\pi^{-1}(\operatorname{Sing} Z)$ only at the point $\Gamma \cap \pi^{-1}(x)$.

The image $\pi(\Gamma)$ is a local holomorphic curve in $Z$. Let $\Delta$ be the unit disk in $\mathbb{C}$. By replacing $\Gamma$ by a relatively compact open neighborhood of $\Gamma \cap \pi^{-1}(x)$ in $\Gamma$, we may assume that there is a nomalization $\sigma: \Delta \longrightarrow \pi(\Gamma)$ of $\pi(\Gamma)$ which is one to one and $\sigma(0)=x$.

We take a positive integer $N$ such that $d / N<\varepsilon_{d-1}-\varepsilon(d)$. By Lemma 3.1 , replace $\Delta$ with a smaller disk if necessary, there exist a positive integer $m$ and a finite number of holomorphic sections $\left\{\widetilde{\tau}_{j}\right\}_{j=1}^{K_{d}} \subset H^{0}\left(X_{1} \times\right.$ $\left.\Delta, p r_{X}^{*} L^{\otimes m(N+1)}\right)$ such that

$$
\left.\tau_{j, u}\right|_{Z} \in H^{0}\left(Z, L^{\otimes m(N+1)} \otimes \mathcal{M}_{Z, \sigma(u)}^{m N}\right) \quad \text { for every } u \in \Delta-0
$$

and for every $j$, and that

$$
x \in X_{0} \cap \bigcap_{j=1}^{K_{d}}\left(\tau_{j}\right)_{0} \varsubsetneqq Z,
$$

where $\tau_{j, u}:=\left.\widetilde{\tau}_{j}\right|_{X_{1} \times u}\left(\right.$ we regard $\left.\tau_{j, u} \in H^{0}\left(X_{1}, L^{\otimes m(N+1)}\right)\right)$ and $\tau_{j}:=\tau_{j, 0}$.

Then we take a positive rational number $\varepsilon$ such that $\varepsilon m r_{0}(1+N)+$ $d / N<\varepsilon_{d-1}-\varepsilon(d)$. For every $u \in \Delta$ and for every $t \geq 0$, we set

$$
\begin{aligned}
\mathcal{I}(u, t) & :=\mathcal{L}^{2}\left(\mathcal{O}_{X_{1}},\left(\sum\left|s_{i}^{(d)}\right|^{2}\right)^{-(1-\varepsilon)}\left(\sum\left|\tau_{j, u}\right|^{2}\right)^{-t /(m N)}\right) ; \\
\alpha(u) & :=\sup \left\{t \geq 0 ; \mathcal{I}(u, t)_{\sigma(u)}=\mathcal{O}_{X, \sigma(u)}\right\} .
\end{aligned}
$$

We see that every $\alpha(u)$ is a positive rational number and that

$$
x \in\left(V \mathcal{I}(0, \alpha(0)) \cap X_{0}\right) \varsubsetneqq Z \subset f^{-1}(f(x)) .
$$

We would like to estimate $\alpha(0)$ by $d$ and $O(\varepsilon)$ (Lemma 4.6 below). The following semicontinuity lemma of multiplier ideal sheaves due to Angehrn and Siu is the key step to reduce the case $x \in \operatorname{Sing} Z$ to the case $x \in \operatorname{Reg} Z$.

LemMa 4.4. ([AS Lemma 6.1]) Let $t_{0}$ be a positive number. Assume that $\alpha(u)<t_{0}$ for almost all $u \in \Delta-0$ with respect to the 2-dimensional Lebesgue measure on $\Delta$. Then $\alpha(0) \leq t_{0}$ holds. 
The outline of the proof is as follows: Assume that $\alpha(0)>t_{0}$. Then the following theorem of Ohsawa and Takegoshi shows that $\alpha(u) \geq t_{0}$ for almost all $u \in \Delta-0$ which is a contradiction.

OHSAWA-TAKegoshi's $L^{2}$-EXTENSION THEOREM 4.5. ([OT]) Let $\Omega$ be a bounded pseudoconvex domain in $\mathbb{C}^{n+1}$ with coordinate $z_{1}, \ldots, z_{n}, w$. Let $H$ be a complex hyperplane defined by $w=0$, and let $\phi$ be a plurisubharmonic function on $\Omega$. Then there exists a constant $C_{\Omega}$ depending only on the diameter of $\Omega$ such that; for any holomorphic function $f$ on $\Omega \cap H$ satisfying

$$
\int_{\Omega \cap H}|f|^{2} e^{-\phi} d V_{n}<\infty
$$

where $d V_{n}$ denotes the $2 n$-dimensional Lebesgue measure, there exists a holomorphic function $F$ on $\Omega$ satisfying $\left.F\right|_{\Omega \cap H}=f$ and

$$
\int_{\Omega}|F|^{2} e^{-\phi} d V_{n+1} \leq C_{\Omega} \int_{\Omega \cap H}|f|^{2} e^{-\phi} d V_{n}
$$

Then we have

LEMMA 4.6. $\alpha(0) \leq d+m N r_{0} \varepsilon$.

Proof. By Lemma 4.4, it is enough to estimate $\alpha(u)$ for $u \in \Delta-0$. We show $\left(\sum\left|s_{i}^{(d)}\right|^{2}\right)^{-(1-\varepsilon)}\left(\sum\left|\tau_{j, u}\right|^{2}\right)^{-t /(m N)}$ is not locally integrable at $\sigma(u)$ for $t \geq d+m N r_{0} \varepsilon$.

We take $u \in \Delta-0$ and a point $\widetilde{x}$ in $\pi^{-1}(\sigma(u)) \cap \Gamma$. We see $\widetilde{x} \in E_{0}$ and $\tilde{x} \notin \bigcup_{i \neq 0} E_{i}$. Let $W$ be an open neighborhood of $\widetilde{x}$ in $\tilde{X}-\bigcup_{i \neq 0} E_{i}$ so that a local coordinate $z, w_{1}, \ldots, w_{n-1}$ on $W$ with $E_{0} \cap W$ defined by $\{z=0\}$, where $n$ is the dimension of $X$. Since $\pi$ maps the $(n-1)$ dimensional manifold $E_{0}$ onto the irreducible $d$-dimensional subvariety $Z$, it follows that the codimension of $\pi^{-1}(x) \cap E_{0}$ in $E_{0}$ is at most $d$ at $\widetilde{x}$. The restriction $\left.\left(\pi^{*} \tau_{j, u}\right)\right|_{E_{0}}$ vanishes to order at least $m N$ at $\widetilde{x}$. Let $\operatorname{Jac}(\pi)$ be the holomorphic Jacobian determinant of the map $\pi$. On $W$ the divisor of $\operatorname{Jac}(\pi)$ is presicely $b_{0} E_{0}$. To conclude the local non-integrability of $\left(\sum\left|s_{i}^{(d)}\right|^{2}\right)^{-(1-\varepsilon)}\left(\sum\left|\tau_{j, u}\right|^{2}\right)^{-t /(m N)}$ at $\sigma(u)$, it suffices to prove the local non-integrability of

$$
|z|^{-2(1-\varepsilon) r_{0}}\left(\sum\left|\pi^{*} \tau_{j, u}\right|^{2}\right)^{-t /(m N)}|z|^{2 b_{0}} \quad \text { at } \widetilde{x} .
$$

Since $r_{0}-b_{0}=1$, by Lemma 3.2, $t \geq d+m N r_{0} \varepsilon$ implies the non-integrability. Hence $\alpha(u)<d+m N r_{0} \varepsilon$ for $u \in \Delta-0$. 
We set $\mathcal{I}(t):=\mathcal{I}(0, t)$ and $\alpha:=\alpha(0)$. Then by Lemma 4.6 , we see $\left(d+\varepsilon_{d-1}\right)-\alpha(1+1 / N)>\varepsilon(d)$. We set the multivalued holomorphic section

$$
\left\{s_{i}^{(d-1)}\right\}_{i=1}^{k_{d-1}}:=\left\{s_{i}^{(d)(1-\varepsilon)} \times \tau_{j}^{\alpha /(m N)} \times t_{k}^{\varepsilon\left(m_{d}+\varepsilon_{d}\right)+\left(d+\varepsilon_{d-1}\right)-\alpha(1+1 / N)}\right\}_{i, j, k}
$$

of $L^{\otimes\left(m_{d-1}+\varepsilon_{d-1}\right)}$ on $X_{1}$. We can verify $(*)_{d-1}$ by noting that $\mathcal{I}_{d-1}(t) \supset$ $\mathcal{I}(t \alpha)$ for $0 \leq t \leq 1$ and that $\mathcal{I}_{d-1}(1)=\mathcal{I}(\alpha)$, where

$$
\mathcal{I}_{d-1}(t):=\mathcal{L}^{2}\left(\mathcal{O}_{X_{1}},\left(\sum\left|s_{i}^{(d-1)}\right|^{2}\right)^{-t}\right)
$$

\section{C. Completion of the proof}

In $4 \mathrm{~A}$ and $4 \mathrm{~B}$, we showed that $(*)_{0}$ hold. Therefore there exist a rational number $\varepsilon_{0}$ with $0<\varepsilon_{0}<1$ and a finite number of multivalued holomorphic sections $\left\{s_{i}^{(0)}\right\}_{i=1}^{k_{0}}$ of $L^{\otimes\left(m_{0}+\varepsilon_{0}\right)}$ on $X_{1}$ such that $x$ is isolated in $V \mathcal{L}^{2}\left(\mathcal{O}_{X_{1}},\left(\sum\left|s_{i}^{(0)}\right|^{2}\right)^{-1}\right)$. We take a smooth Hermitian metric $h_{1}$ of $\left.L\right|_{X_{1}}$ such that the curvature form gives a complete Kähler form on $X_{1}$. Then we get the desired singular Hermitian metric

$$
H_{m}:=h_{1}^{m-\left(m_{0}+\varepsilon_{0}\right)} \frac{h_{1}^{m_{0}+\varepsilon_{0}}}{\sum\left|s_{i}^{(0)}\right|_{1}^{2}}
$$

for every integer $m>m_{0}=d_{0}\left(d_{0}+1\right) / 2$, where $\left|s_{i}^{(0)}\right|_{1}$ is the length with respect to $h_{1}$.

\section{REFERENCES}

[AS] U. Angehrn, Y. T. Siu, Effective freeness and point separation for adjoint bundles, Invent. math., 122 (1995), 291-308.

[D1] J. P. Demailly, A numerical criterion for very ample line bundles, Jour. of Diff. Geom., 37 (1993), 323-374.

[D2] J. P. Demailly, $L^{2}$ vanishing theorem for positive line bundles and adjunction theory, to appear, CIME Session, Transcendental Methods in Algebraic Geometry, Cetraro, Italy (1994).

[F] T. Fujita, On polarized manifolds whose adjoint bundles are not semipositive, Algebraic Geometry, Sendai, 1985, Advanced Studies in Pure Math., 10, North-Holland, Amsterdam (1987), pp. 167-178.

[K] Y. Kachi, Extremal contractions from 4-dimensional manifolds to 3-folds, preprint (1995). 
[KMM] Y. Kawamata, K. Matsuda, K. Matsuki, Introduction to the Minimal Model Problem, Algebraic Geometry, Sendai, 1985, Advanced Studies in Pure Math., 10, North-Holland, Amsterdam (1987), pp. 283-360.

[L] Lazarsfeld R., Lectures on Linear Series, to appear (1994).

[N] A. M. Nadel, Multiplier ideal sheaves and existence of Kähler-Einstein metrics of positive scalar curvature, Ann. of Math., 132 (1990), 549-596.

[OT] T. Ohsawa, K. Takegoshi, On the Extension of $L^{2}$ Holomorphic Functions, Math. Z., 195 (1987), 197-204.

[Tj] H. Tsuji, Global generation of adjoint bundles, Nagoya Math. J., 142 (1996), 5-16.

Department of Mathematics

Naruto University of Education

Takashima, Naruto-cho, Naruto-shi, 772

Japan

takayama@naruto-u.ac.jp 